Tropical Journal of Pharmaceutical Research April 2017; 16 (4): 803-810

ISSN: $1596-5996$ (print); 1596-9827 (electronic)

(C) Pharmacotherapy Group, Faculty of Pharmacy, University of Benin, Benin City, 300001 Nigeria.

All rights reserved.

Available online at http://www.tjpr.org

Original Research Article

http://dx.doi.org/10.4314/tjpr.v16i4.9

\title{
Septin 9 hypermethylation contributes to migration and resistance to drug treatments in colon cancer
}

\author{
Tao Peng ${ }^{1,2}$, Fanguo Kong ${ }^{2}$, Xingyuan Diao ${ }^{2}$, Jiaguo Huang ${ }^{2}$ and Xishuang Liu ${ }^{1 *}$ \\ ${ }^{1}$ Department of Gastroenterology, Affiliated Hospital of Qingdao University, Qingdao, Shandong, 266000, ${ }^{2}$ Department of \\ Gastroenterology, The People's Hospital of Laiwu City, Laiwu, Shangdong, 271199, China
}

*For correspondence: Email: xishuangliu@hotmail.com; Tel/Fax: 0086-0532-82911219

\begin{abstract}
Purpose: To examine septin 9 gene-promoter methylation content in colorectal cancer and establish its significance in cancer progression and chemoresistance.

Methods: Patient samples and colorectal cancer cell lines (CRC) were evaluated for septin 9 expression and promoter hypermethylation content. Septin 9 promoter methylation and expression in cells were perturbed by 5-AZA (5-aza-2'-deoxycytidine) treatments or overexpression and probed for changes in Rho A signaling, cell proliferation, and migration. Finally, the significance of septin 9 methylation in chemoresistance was probed using apoptotic assays in CRC cells and in a xenograft tumor model.

Results: Expression analysis showed a reduction in septin 9 levels in tumor tissues $(p<0.001)$ and cell lines $(p<0.01)$, while an increase in septin 9 promoter methylation was seen, respectively ( $>2$-fold; $p<$ 0.01). Increasing septin 9 levels in CRC cells by 5-AZA treatments or overexpression showed decreased Rho A signaling and cell migration $(p<0.01)$, whereas cell proliferation remained unaffected. Furthermore, increasing septin 9 levels also exhibited increased cisplatin-induced apoptosis in CRC cells and reduced chemoresistance in the mouse ( 2-fold; $p<0.01)$.

Conclusion: Septin 9 promoter hypermethylation reduces septin 9 expression and promotes migration and chemoresistance.
\end{abstract}

Keywords: Septin 9, Hypermethylation, Colorectal cancer, Drug resistance, Rho A signaling

\begin{abstract}
Tropical Journal of Pharmaceutical Research is indexed by Science Citation Index (SciSearch), Scopus, International Pharmaceutical Abstract, Chemical Abstracts, Embase, Index Copernicus, EBSCO, African Index Medicus, JournalSeek, Journal Citation Reports/Science Edition, Directory of Open Access Journals (DOAJ), African Journal Online, Bioline International, Open-J-Gate and Pharmacy Abstracts
\end{abstract}

\section{INTRODUCTION}

Colorectal cancer (CRC) is one of the leading cancer types causing mortality worldwide [1]. Treatment options such as surgical resection, radiotherapy and chemotherapy are widely used but its outcomes are largely based on the stage of cancer $[2,3]$. The patients with stage I CRC exhibit $94.1 \%$ five-year survival rate while it is significantly reduced to $24 \%$ in stage IV disease $[2,3]$. Thus early detection of the cancer has a significant impact on the treatment outcome.
Changes in DNA methylation profile of critical genes in cells is one of the early molecular changes in cancer progression [4,5]. These epigenetic events combined with diseasecausing mutations provide a selective advantage for cancer initiation and progression. Multiple studies have shown that septin 9 gene spanning a region of $219 \mathrm{~kb}$ in the genome and encoding different isoforms is epigenetically modified in the promoter region, and can be used as a diagnostic test for CRC [6-8].

Recent studies have shown that septin 9 promotes angiogenesis and facilitate tumor 
growth in the hetero-transplant model [9]. In contrast, breast cancer-related studies have indicated distinct cellular functions and suggested a tumor-suppressor role [10]. Despite that, it remains unclear how septin 9 hypermethylation affects gene expression in normal and neoplastic colon tissues and cells, and the underlying molecular mechanisms triggered due to aberrant septin 9 methylation in tumor development and chemoresistance, particularly colorectal cancer (CRC).

\section{EXPERIMENTAL}

\section{Cells and tissue samples}

SW480, CCD-18Co, HT-29, HS6751, HCT-116 and LoVo cell lines were purchased from American Type Culture Collection (ATCC) and maintained as per the instructions provided. For patient samples, colon neoplastic and adjacent non-transformed tissue samples were obtained from institute's pathology archive following institutional review board approval from The People's Hospital of Laiwu City, Laiwu, Shangdong (Protocol no. 2705S). Tissue samples were then processed accordingly for different procedures.

\section{Quantitative realtime polymerase chain reaction (qRT-PCR)}

Total RNA was extracted from cells and tissue samples using RNAeasy kit (Qiagen) and cDNA synthesis was performed as described previously [11]. Gene-specific PCR was performed CFX96

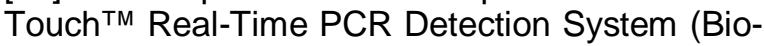
Rad, USA) using specific TaqMan probes and universal PCR Master Mix (Life Technologies, USA). The TaqMan probes used for analyzing the gene expressions were: Septin 9Hs00246396_m1; GAPDH -Hs02758991_g1 (Applied Biosystems, USA)

\section{Pyrosequencing assays targeting Septin 9 promoter regions}

DNA was extracted from cells and patient samples and evaluated for the status of methylation in $\mathrm{CpG}$ dinucleotides located in septin 9 promoter region. We utilized the Qiagen PyroMark assay kit (Qiagen, USA) and investigated the methylation status as described previously [12 ] using the primer sets: Fwd: GATCTAGCCTAGGGTTCCAG, Rev: ACCGTTCGTGCAGACGAG (5'-biotin labeled) and the sequencing probe: TTGACGCGGTGCTAG. Briefly, the PCR products were suspended using the PyroMark Q24 (Qiagen) following the manufacturer's protocol, and the methylation status was quantified with an estimated score between 0100 with 0 being no methylation and 100 represents complete methylation for all of the $\mathrm{CpG}$ dinucleotide in the region. The threshold in the assay was $>5 \%$.

\section{Immunoblotting}

Total cell lysates were prepared using RIPA buffer and proteins were separated on a $4-15 \%$ SDS-PAGE gel under denaturing conditions. Proteins were then transferred to PVDF membrane, blocked and probed for specific proteins using antibodies: Septin 9 (1: 1000, Novus Biologicals, USA); Actin (1:2000, Cell Signaling Technology, USA); Rho A (1: 500, Cell Signaling Technology, USA). The membranes were then incubated with secondary antibodies conjugated with horseradish peroxidase followed by chemiluminescence substrate based development (Super Signal West Dura, Pierce Biotechnology, USA).

\section{Cell proliferation assays}

Cell proliferation assays were performed using Alamar Blue assay (Invitrogen, USA) [11]. Briefly, control or overexpressing cells $\left(1 \times 10^{4}\right.$ per well) were seeded in a 96-well plate and after $16 \mathrm{~h}, 50$ $\mu \mathrm{L}$ of Alamar reagent $(1 \mathrm{mg} / \mathrm{mL}$ ) was added and incubated for $2 \mathrm{~h}$. Change in Alamar blue oxidation is noted as a measure of cell density. These values were noted at different time points as indicated. Optical density values were noted at $570 \mathrm{~nm}$ using a Microplate reader and proliferation trend over time was plotted.

\section{Cell migration assays}

Cell migrations assays were performed using a chemotaxis chamber (NeuroProbe, Gaithersburg, MD) [13]. Briefly, cells were seeded in the upper chemotaxis chamber and allowed to migrate for $24 \mathrm{~h}$. After scraping the non-migrated cells from the top side, the cells that are migrated to the bottom side were stained using Diff-Quik Stain Set (Dade Behring, Eschborn, Germany) and quantified. At least five independent fields were randomly picked per sample and quantified.

\section{Immunohistochemistry}

Tissue sections were deparaffinized with xylene and gradient ethanol washes, rehydrated, and antigen retrieved in sodium citrate buffer $(\mathrm{pH}$ 6.0). Tissue sections were then blocked with $5 \%$ BSA and $0.2 \%$ goat serum. Primary antibodies specific to septin 9 (Novus Biologicals, USA) were incubated $(1: 50 \mathrm{v} / \mathrm{v})$, followed by secondary 
antibody (1:100) and counterstained for the nucleus.

\section{Overexpression experiments}

For overexpression, the coding DNA sequence of septin 9_v 1 isoform was cloned into pcDNA3.1 and transfected into cells. Stable cells were selected with geneticin selection (G418-2 $\mu \mathrm{g} / \mathrm{mL}$; Invitrogen).

\section{Drug treatments}

For cell-based experiments, cells were treated with cisplatin $(5 \mu \mathrm{M}) 24 \mathrm{~h}$ and evaluated for apoptosis by TUNEL assay. For mouse experiments, BALB/c (nu/nu) mice were inoculated with SW480 or LoVo cells $\left(2.5 \times 10^{6}\right.$ per mouse) subcutaneously and administered with cisplatin ( $5 \mu \mathrm{M} /$ mouse) intraperitoneally (i.p.) on days $3,6,10,14,20$. As the mice used in the study ranged in similar weight we used a same dose ( $5 \mu \mathrm{M} /$ mouse) of injection. Tumor size was shown as long diameter time short diameter $\left(\mathrm{mm}^{2}\right)$. All mouse experiments were performed with prior approval from the institute's animal care committee at The People's Hospital of Laiwu City, Laiwu, Shangdong (protocol no: 251216). Housing and all procedures using animals were performed in accordance with the Animals (Scientific Procedures) Act, 1986 (UK) (amended 2013) and reported as per the ARRIVE Guidelines for reporting animal research [14]. All mice were housed in individually ventilated cages (5 per cage) under specific pathogen-free (SPF) condition, temperaturecontrolled, and in a $12 \mathrm{~h} / 12 \mathrm{~h}$ light/dark cycle. Animal weights were routinely monitored every other day.

\section{Detection of apoptosis}

Apoptosis was detected using a TUNEL staining kit (Promega, WI) [15]. TUNEL staining was performed directly in cells and an absolute number of TUNEL positive cells in randomly picked microscopy fields were noted.

\section{Pulldown assay}

RhoA activity in cell lysates was measured using Rho-binding pull-down assay as per the manufacturer's instructions (EMD Millipore, Germany). The ratio of guanosine triphosphate (GTP-bound) to total Rho was measured and used as an index for its activity.

\section{Statistical analysis}

Data were analyzed using one-way ANOVA with statistical significance defined as $p<0.05$. All analyses were performed using GraphPad Prism (version 6) and all values are listed as mean \pm standard deviation (SD).

\section{RESULTS}

\section{Septin 9 expression in colon cancer tissues and cell lines}

To begin with, we evaluated the expression of septin 9 in primary colon cancer tissues with their corresponding adjacent normal tissue regions as respective controls. The results as evaluated through immunostaining showed an absence or decreased expression of septin 9 in tumor tissues as compared to normal regions (Figure 1A). In addition, septin 9 mRNA analysis using PCR showed a similar decrease in septin 9 expressions in tumor tissues ( 58\%; $p<0.001)$ (Figure 1B). While, these results confirmed the septin 9 decrease in tumor samples, CRC cell lines showed a varied expression profile for Septin 9 in different cell lines. Our results in CRC lines as evaluated through septin 9 specific PCR showed that maximum decrease was seen in the most invasive cell line such as SW480 and LoVo cells $(\sim 50 \% ; p<0.01)$ while in other cell lines tested septin 9 mRNA were reduced but to varying levels (Figure $1 \mathrm{C}$ ).

\section{Septin 9 hypermethylation causes reduced expression in tumor tissues and cell lines}

To determine whether methylation status of the septin 9 promoter is involved in the decreased expression of septin 9 in tumor tissues and cell lines, we first evaluated the promoter methylation using a pyrosequencing assay targeting the promoter region of septin 9. The results showed an increased methylation in the septin 9 promoter region in tumor tissues compared to the adjacent normal tissues ( $>$ 3fold; $p<0.01$ ) (Figure 2A). Similarly, SW480 and LoVo cells exhibited hypermethylation in the promoter regions, compared to other cell lines (> 2.5 fold; $p<0.01)$. In addition, 5-AZA (5-aza-2'deoxycytidine), a demethylation agent, treatments of SW480 and LoVo cells increased septin 9 expression (Figure 2C). These results together indicate that hypermethylation of septin 9 promoter regions negatively regulates septin 9 expression. 
A.

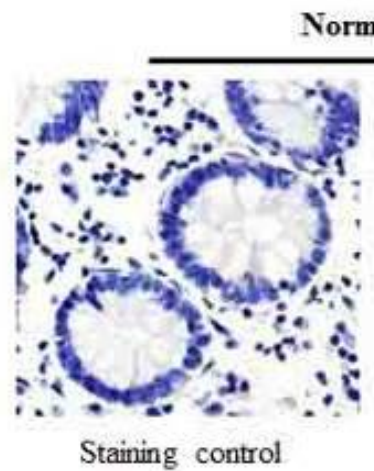

Normal Colon

CRC

B.
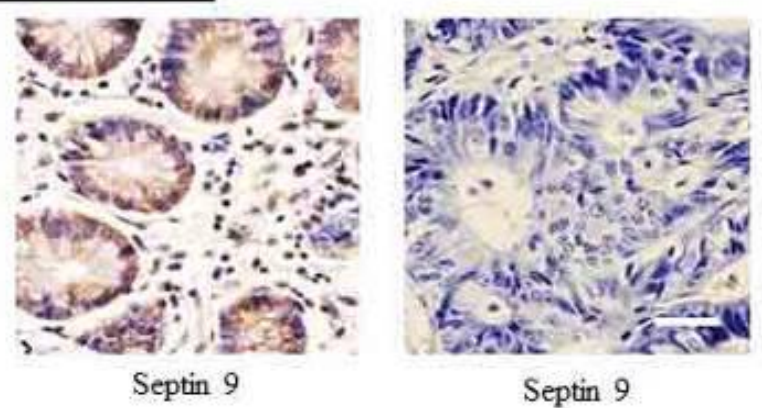

Septin 9

c.
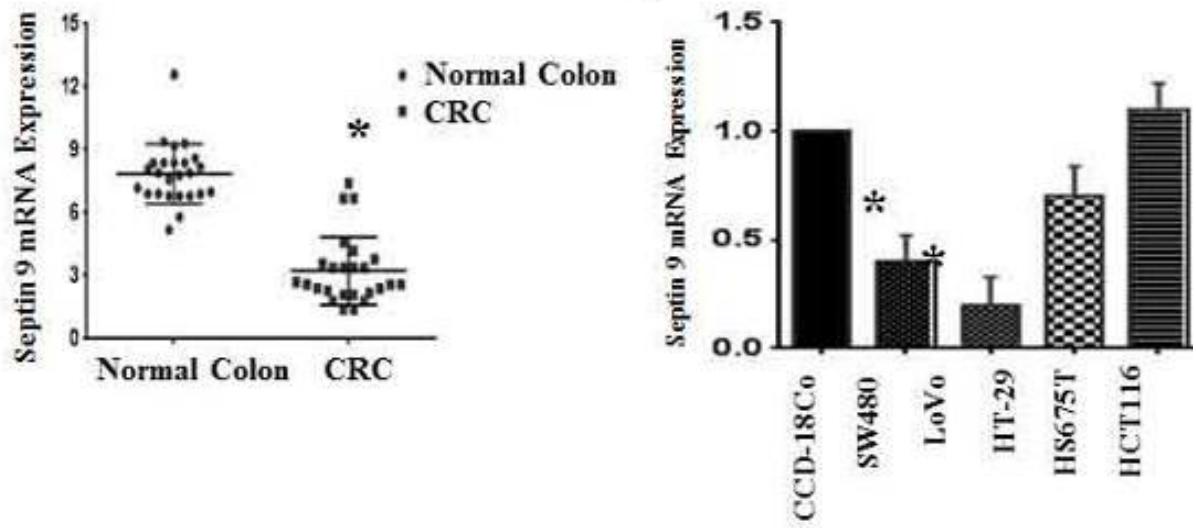

Figure 1: Septin 9 expression in colon cancer patient samples and cell line

(A) Septin 9 expression levels were evaluated by immunostaining in primary tissue samples. Representative cases are shown (40X). Normal colon cells exhibited expression of septin 9, while it is minimal or absent in carcinoma samples. Scale bar $5 \mu \mathrm{M}$. (B) Septin $9 \mathrm{mRNA}$ expression profiles in patient samples. Expression levels from all the 25 CRC region specimens and matched normal colon region specimens were quantified by $q R T-P C R$. Septin 9 expression was significantly reduced in cancerous regions as compared to normal regions (8.1 \pm 0.95 fold in normal regions versus $3.4 \pm 1.72$ in cancer, $\left.{ }^{*} p<0.001\right)$. (C) Septin 9 mRNA expression profiles in CRC cell lines. SW480 and LoVo cells showed a significant reduction compared to normal cells (CCD18Co) $\left({ }^{*} p<0.01\right)$

A.

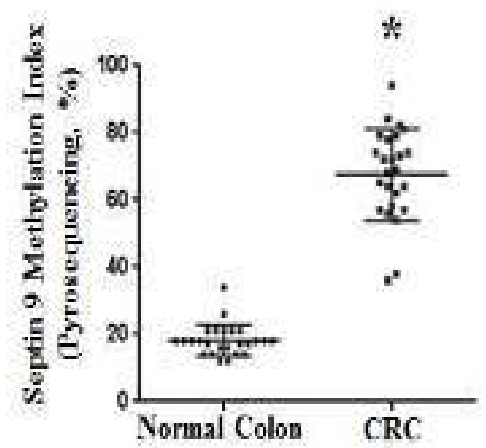

B.

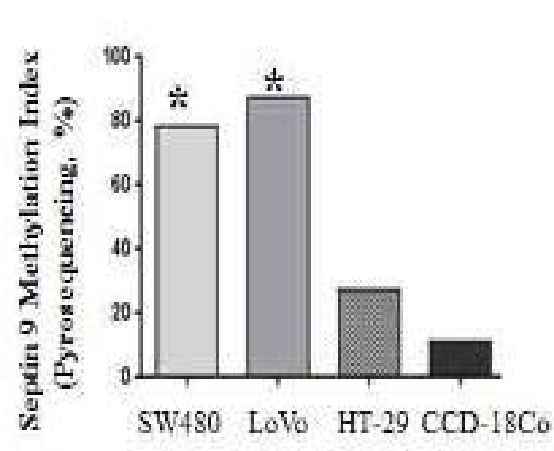

c.

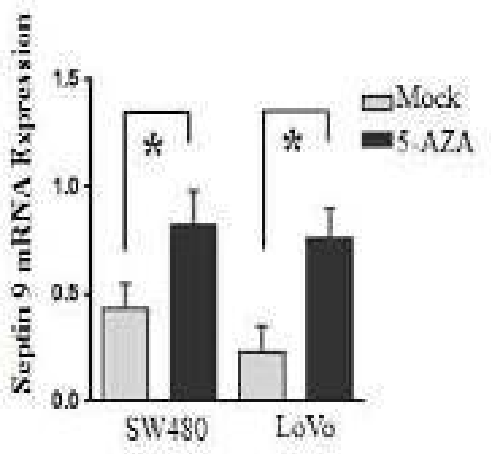

Figure 2: DNA methylation status inversely correlates with loss of septin 9 expression in CRCs. (A). The septin 9 promoter regions are highly methylated in CRC patients as opposed to normal colon regions $\left({ }^{*} p<0.001\right)$. (B) SW480 and LoVo cell lines exhibited the high methylation in promoter regions of septin 9, whereas the HT-29 and CCD-18Co (normal) cells showed minimal promoter methylation. $\left({ }^{*} p<0.01\right)$ (C) $5-A Z A$ treatments in SW480 and LoVo cell lines led to significantly increased septin 9 levels. Values were normalized against baseline expression in CCD-18Co cells ( ${ }^{*} p$ $<0.01)$ 


\section{Septin 9 suppresses colorectal cancer migration, but not proliferation in vitro}

To understand the consequence of hypermethylation induced decreased septin 9 expressions in cancer progression, we constitutively overexpressed the septin 9 in SW480 cells and LoVo cells ( $50 \%$ reduced; $p$ $<0.01$ ) (Figure 3A) and evaluated their proliferation and migration. The result showed that septin 9 overexpression significantly decreased migration for both the cell types with septin 9 overexpression, while no significant change in their cell proliferation rate in vitro (Figure 3B and Figure3C) were noted. In addition, as septin 9 is modulating migration in both the cell types, which is usually regulated by GTPases, we chose to evaluate for changes in Rho A. The results showed that septin 9 overexpression significantly decreased the Rho A activation pathway as determined by reduced RhoA-GTP levels as compared to total Rho A levels $(\sim 80 \%$ reduced; $p<0.01)$. These results suggest that septin 9 suppresses cell migration by negatively modulating the Rho A pathway (Figure 4).
A.

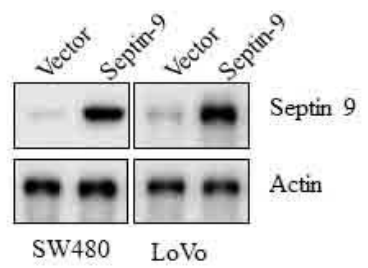

B.

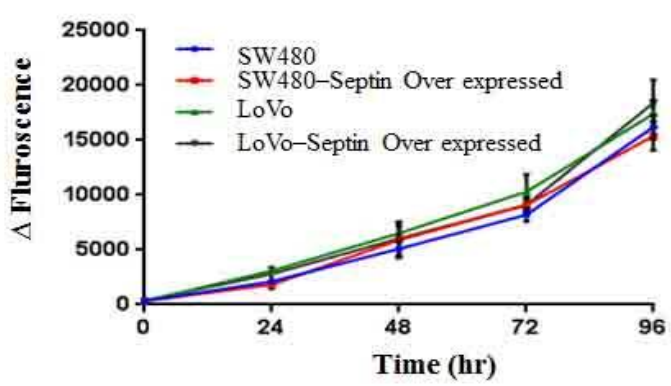

C.

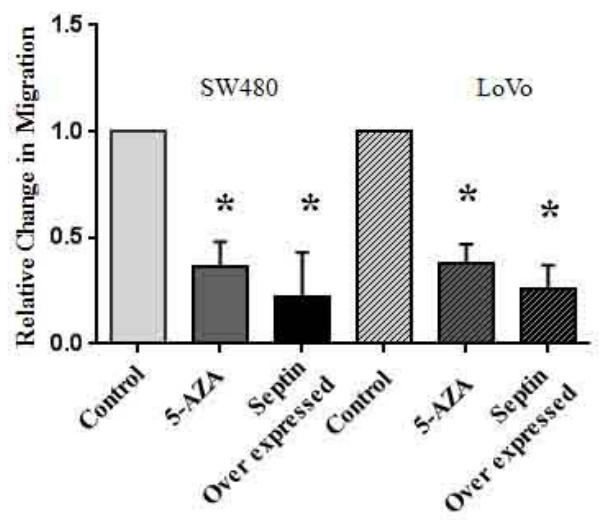

Figure 3: Septin 9 overexpression correlates with decreased migration but not proliferation

(A) Stable cells of both SW480 and LoVo cells overexpressing septin 9 were prepared. Immunoblot of septin 9 and actin was shown. (B) Alamar blue assay showing no change in proliferation with overexpressing septin 9. (C) Decrease in migration were noted in both SW480 and LoVo cells upon overexpressing septin 9 or with 5-AZA treatment $\left({ }^{*} p<0.01\right)$
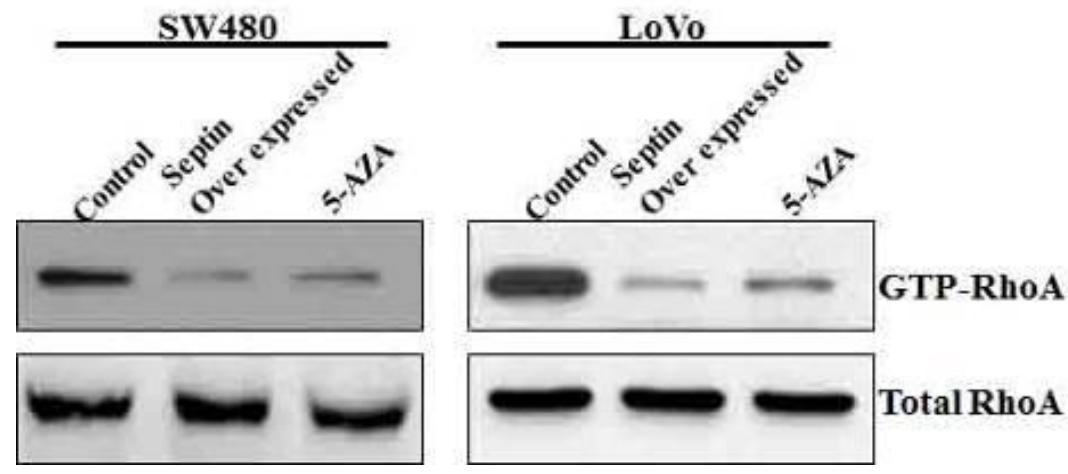

Figure 4: Septin 9 expression levels are associated with altered RhoA signaling.

Increased Rho-GTP was present in SWSW480 cells and LoVo cells, and is reduced with overexpression of septin 9 or $5-A Z A$ treatments $(\sim 80 \%$ reduced; $p<0.01)$. Representative immunoblots are shown 
A.
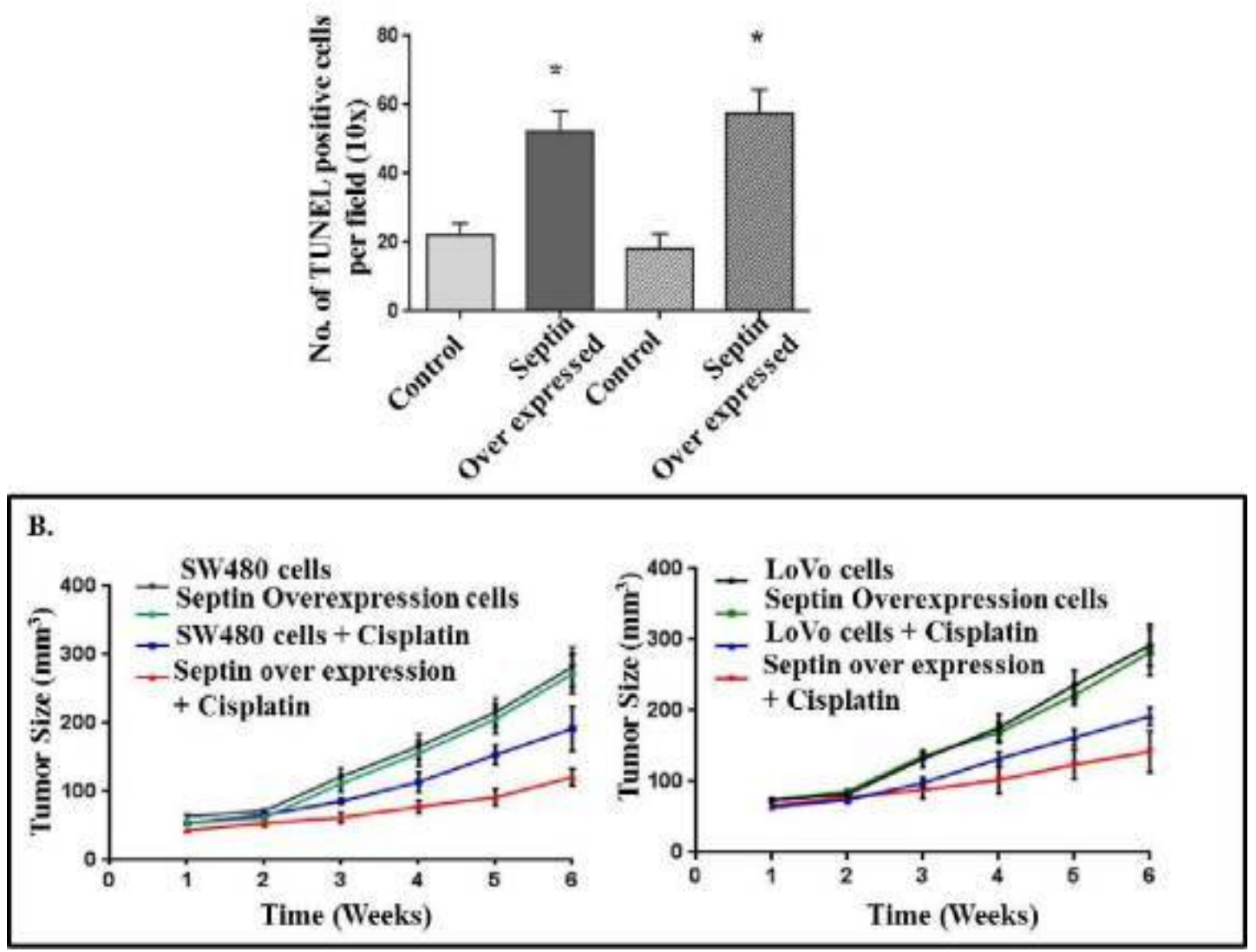

Figure 5: Septin 9 might contribute to drug-induced apoptosis in vitro and in vivo

(A) SW480 cells and LoVo cells with or without overexpression of septin 9 were treated with cisplatin (5 $\mu$ M) and evaluated for apoptosis. TUNEL positive staining was quantified. Overexpression of septin 9 enhances apoptosis $\left({ }^{*} P<0.05\right)$ (B) Xenograft models of SW480 and LoVo cells with or without septin 9 overexpression were established and treated with cisplatin. Changes in tumor size were measured over time. Septin overexpression enhanced the effect of cisplatin-induced reduction in tumor size in both cell types from third week onwards ( ${ }^{*}<<$ 0.01)

Septin 9 expressions contribute to druginduced apoptosis in vitro and in vivo

We evaluated the efficacy of cisplatin in SW480 and LoVo CRC cells with the over-expression of septin 9. The results showed an increased druginduced apoptosis as measured by TUNEL assay in overexpressing cells than control cells (> 2 fold enhanced; $p<0.05$ ) (Figure 5A). In addition, overexpression of septin 9 significantly enhanced the drug efficiency in a xenograft tumor model ( 2 fold enhanced; $p<0.01$ ). These increased efficiencies were correlating with increased TUNEL positive cells in septin 9 overexpressing cells (Figure 5B).

\section{DISCUSSION}

It is well established now that DNA methylation in the genome could modulate gene expression [16]. In particular, multiple CpG-rich promoter regions were extensively studied over the last decade and associated the methylation content with effect on transcription $[17,18]$. Similar hypermethylation content was also previously identified in promoter regions of septin 9 and implicated for modulating expression in breast cancer cells [19]. In the current study, we observed that septin 9 expressions in tissues correlate with methylation content in the promoter regions in CRC patient samples. This notion is further supported by the decreased septin 9 expressions in colon cancer cells that are hypermethylated in promoter regions. In addition, we have shown that demethylation treatment restored at least partially the expression levels of septin 9, thereby suggesting that expression regulation in tumor conditions is achieved by aberrant mode of epigenetic modifications.

Since septin 9 is involved in modulating cytoskeleton [20], it is conceivable that suppression of septin 9 could modulate multiple 
related processes. In accordance with this notion, other studies have also shown a function of septin 9 in multiple processes such as migration and cilia formation. Our data have shown that suppressed septin 9 expression facilitate migration and altered Rho A signaling, while proliferation remains unaffected. This suggests that septin 9 functions are contextdependent, and identification of other binding proteins or other means of context could aid us to understand septin 9 mediated effects in these processes further.

Multiple studies have highlighted increased resistance to chemotherapy in advanced CRC patients $[21,22]$. Given that septin 9 suppression is one of the early biomarker and prerequisite for CRC progression, we reasoned, if this would facilitate the development of chemoresistance in later stages. Our data have shown that septin 9 overexpression in cells increases efficiency in drug-induced apoptosis. While the specific molecular mechanism through which septin 9 modulates drug efficiency needs further investigation, similar proteins such as septin 4 effects in hepatocellular carcinoma suggests the involvement of increased expression of p21 and decrease in pro- caspases such as pro-caspase 7 and 3 [23].

Together, our data indicate that septin 9 plays a role in tumor suppression and the particular mode of its regulation such as hypermethylation affects its expression and related functions. However, it also worth mentioning that some of the earlier evidence have highlighted oncogenic function for septin 9 in breast cancer cells and attributed it to the different isoforms of septin 9 expression and localization. An intriguing question raised by our study in the context of these data is, how septin 9 hypermethylation contributes to variations in isoform expression in colon tissues, and how the different isoforms would affect CRC progression. While we have limited our analysis to septin 9_v1 isoform, future studies aimed at understanding these different isoform expression patterns in colon tissues and its relation to hypermethylation content and chemoresistance would provide more insights into these complex processes.

\section{CONCLUSION}

The findings of this study provide the first set of evidence for hypermethylation-induced reduction of septin 9 expression, which facilitates the migration and invasion of CRC cells and development of chemoresistance. Thus, chemotherapy options with concurrent treatments to increase septin 9 expression may improve outcomes in colorectal cancers.

\section{DECLARATIONS}

\section{Acknowledgement}

None declared.

\section{Conflict of Interest}

No conflict of interest associated with this work.

\section{Contribution of Authors}

The authors declare that this work was done by the authors named in this article and all liabilities pertaining to claims relating to the content of this article will be borne by them.

\section{Open Access}

This is an Open Access article that uses a funding model which does not charge readers or their institutions for access and distributed under the terms of the Creative Commons Attribution License (http://creativecommons.org/licenses/by/ 4.0) and the Budapest Open Access Initiative (http://www.budapestopenaccessinitiative.org/rea d), which permit unrestricted use, distribution, and reproduction in any medium, provided the original work is properly credited.

\section{REFERENCES}

1. Siegel $R$, Naishadham $D$, Jemal A. Cancer statistics, 2012. CA Cancer J Clin 2012; 62: 10-29.

2. Sievers $C K$, Kratz JD, Zurbriggen $L D$, LoConte $N K$, Lubner SJ, Uboha N, Mulkerin D, Matkowskyj KA, Deming DA The Multidisciplinary Management of Colorectal Cancer: Present and Future Paradigms. Clin Colon Rectal Surg. 2016; 29(3): 232-238.

3. Zhai Z, Yu X, Yang B, Zhang $Y$, Zhang L, Li X, Sun $H$. Colorectal Cancer Heterogeneity and Targeted Therapy: Clinical Implications, Challenges and Solutions for Treatment Resistance. Semin Cell Dev Biol 2016 Aug 27. pii: S1084-9521(16) 30267-1. doi: 10.1016/j.semcdb. 2016.08.033.

4. Laird PW, Jaenisch $R$. The role of DNA methylation in cancer genetic and epigenetics. Annu Rev Genet 1996; 30:441-464.

5. Judson H, Stewart A, Leslie A, Pratt NR, Baty DU, Steele $R J$, Carey FA. Relationship between point gene mutation, chromosomal abnormality, and tumour suppressor gene methylation status in colorectal adenomas. J Pathol 2006; 210(3): 344-350. 
6. Fung KY, Dai L, Trimble WS. Cell and molecular biology of septins. Int Rev Cell Mol Biol 2014; 310: 289-339.

7. Warren JD, Xiong W, Bunker AM, Vaughn CP, Furtado LV, Roberts WL, Fang JC, Samowitz WS, Heichman $K A$. Septin 9 methylated DNA is a sensitive and specific blood test for colorectal cancer. BMC Med 2011; 9: 133.

8. Tóth K, Sipos F, Kalmár A, Patai AV, Wichmann B, Stoehr R, Golcher H, Schellerer V, Tulassay Z, Molnár $B$. Detection of methylated SEPT9 in plasma is a reliable screening method for both left- and right-sided colon cancers. PLoS One 2012; 7(9): e46000.

9. Amir S, Golan M, Mabjeesh NJ. Targeted knockdown of SEPT9_v1 inhibits tumor growth and angiogenesis of human prostate cancer cells concomitant with disruption of hypoxia-inducible factor-1 pathway. Mol Cancer Res 2010; 8(5): 643-652.

10. Connolly D, Yang Z, Castaldi M, Simmons N, Oktay MH, Coniglio S, Fazzari MJ, Verdier-Pinard $P$, Montagna $C$. Septin 9 isoform expression, localization and epigenetic changes during human and mouse breast cancer progression. Breast Cancer Res 2011; 13(4): R76.

11. Subramanian B, Ko WC, Yadav V, DesRochers TM, Perrone RD, Zhou J, Kaplan DL. The regulation of cystogenesis in a tissue engineered kidney disease system by abnormal matrix interactions. Biomaterials 2012; 33(33): 8383-8394.

12. Cui J, Cai Y, Hu Y, Huang Z, Luo Y, Kaz AM, Yang Z, Chen $D$, Fan $X$, Grady WM, Wang J. Epigenetic silencing of TPM2 contributes to colorectal cancer progression upon RhoA activation. Tumour Biol 2016 Jun 23. [Epub ahead of print]

13. Jacob K, Webber M, Benayahu D, Kleinman HK. Osteonectin promotes prostate cancer cell migration and invasion: a possible mechanism for metastasis to bone. Cancer Res 1999; 59(17): 4453-4457

14. Kilkenny C, Browne WJ, Cuthill IC, Emerson M, Altman $D G$. Improving bioscience research reporting: the
ARRIVE guidelines for reporting animal research. PLOS Biol 2010; 8(6): e1000412

15. Subramanian B, Anand M, Khan NW, Khanna H. Loss of Raf-1 kinase inhibitory protein delays early-onset severe retinal ciliopathy in Cep290rd16 mouse. Invest Ophthalmol Vis Sci 2014; 55(9): 5788-5794.

16. Chhabra R. miRNA and methylation: a multifaceted liaison. Chembiochem 2015; 16(2): 195-203.

17. Abdelfatah E, Kerner Z, Nanda N, Ahuja N. Epigenetic therapy in gastrointestinal cancer: the right combination. Therap Adv Gastroenterol 2016; 9(4): 560-579.

18. Wasserkort R, Kalmar A, Valcz G, Spisak S, Krispin M, Toth K, Tulassay Z, Sledziewski AZ, Molnar B. Aberrant septin 9 DNA methylation in colorectal cancer is restricted to a single CpG island. BMC Cancer 2013; 13: 398.

19. Karabulut S, Kaya Z, Gullu G, Peker I, Özmen T, Gulluoglu BM, Kaya H, Erzik C, Ozer A, Akkiprik M. Correlation between the DNA methylation and gene expression of IGFBP5 in breast cancer. Breast Dis 2016 Aug 11. [Epub ahead of print]

20. Weirich CS, Erzberger JP, Barral Y. The septin family of GTPases: architecture and dynamics. Nat Rev Mol Cell Biol 2008; 9(6): 478-489.

21. Marin JJ, Sanchez de Medina F, Castaño B, Bujanda L, Romero MR, Martinez-Augustin O, Moral-Avila RD, Briz $O$. Chemoprevention, chemotherapy, and chemoresistance in colorectal cancer. Drug Metab Rev 2012; 44(2): 148-172.

22. Yang SY, Sales KM, Fuller B, Seifalian AM, Winslet MC. Apoptosis and colorectal cancer: implications for therapy. Trends Mol Med 2009; 15(5): 225-233.

23. Shen S, Liu M, Wu Y, Saiyin H, Liu G, Yu L. Involvement of SEPT4_i1 in hepatocellular carcinoma: SEPT4_i1 regulates susceptibility to apoptosis in hepatocellular carcinoma cells. Mol Biol Rep 2012; 39(4): 45194526. 